\title{
Clinical use of the hyperbaric oxygen bed
}

\author{
R. ASHFIELD* \\ M.B., B.S., M.R.C.P. \\ Research Senior Registrar, \\ Thoracic Unit, Westminster Hospital
}

\author{
C. E. DREW \\ M.V.O., V.R.D., F.R.C.S. \\ Thoracic Surgeon, \\ Westminster Hospital
}

\section{Summary}

The Vickers hyperbaric oxygen bed is described and details of its use are given in the treatment of 207 patients with a wide variety of clinical conditions. As part of a deliberate policy, a special study was made of its place in the treatment of severe acute myocardial infarction.

No major procedural difficulties were encountered. Results are generally encouraging and frequently of dramatic benefit.

\section{Introduction}

Interest in the clinical use of hyperbaric oxygen was stimulated by the work of Boerema and his colleagues (Boerema, Brummelkamp \& Meijne, 1964) on studies which had been initiated nearly 10 years previously.

There are two methods of using hyperbaric oxygen. The first is to use a chamber the size of a small room. Patients and attendants are subjected to hyperbaric conditions. The advantages of this type of apparatus are that more than one patient can be treated at the same time, and for research purposes the patient or subject may be studied within the chamber using appropriate measuring apparatus and other research equipment. The chamber may also be used as an operating theatre. A high tissue $\mathrm{Po}_{2}$ is achieved by giving oxygen by mask or endotracheal tube.

The second method is to use an individual chamber using pure oxygen. The advantages of the individual chamber are as follows:

(1) There is a considerable saving in installation space and initial cost.

(2) Only one-fifth the pressure of compressed air is required to obtain the same $\mathrm{PO}_{2}$.

(3) Tracheal intubation or a face mask are not required.

(4) Complications from nitrogen cannot occur.

(5) Small hyperbaric oxygen chambers can be installed in hospital side rooms or wards with-

\footnotetext{
* Now physician, Dorset County Hospital, Dorchester.
}

out modification of the existing structure and can be run from the hospital piped oxygen supply or from a cylinder.

(6) Medical and nursing staff are not subjected to hyperbaric conditions.

The main disadvantage of the individual chamber is the fact that the patient is isolated from his attendants and any measurements must be made by apparatus connected to the patient before the chamber is closed, or else by direct observations through glass or Perspex. The patient must be decompressed for nursing care or urgent medical treatment. In practice, premature decompression is rarely required.

This paper concerns our experience in the use of a hyperbaric oxygen bed designed and constructed by Vickers Medical. Preliminary work began in 1962 and the prototype was installed in Westminster Hospital in 1964. Based on experience gained during the following year a new improved model was introduced and has been in continuous use since April 1966.

\section{The apparatus}

The hyperbaric oxygen bed was designed particularly for patients who cannot lie flat for long periods. In it they may sit upright or recline on an adjustable back rest at any angle. Moreover, the entire bed can be tilted head up or down through an angle of $10^{\circ}$ in either direction. Reclining or upright, the patient has an uninterrupted view of his surroundings and he can be clearly seen. There are two plastic observation portholes at the foot end of the bed. At the head end is a plastic dome. The lid of the chamber which includes the dome, opens upwards on a balanced hinge at the foot, and when closed is locked on to a gas-tight seal, converting it into a pressure chamber (Fig. 1). When opened there is a vertical gap of 44 in. $(112 \mathrm{~cm})$ at the head end. An adjacent console contains the valve gear and the closedcircuit oxygen conditioner, and is connected to the 

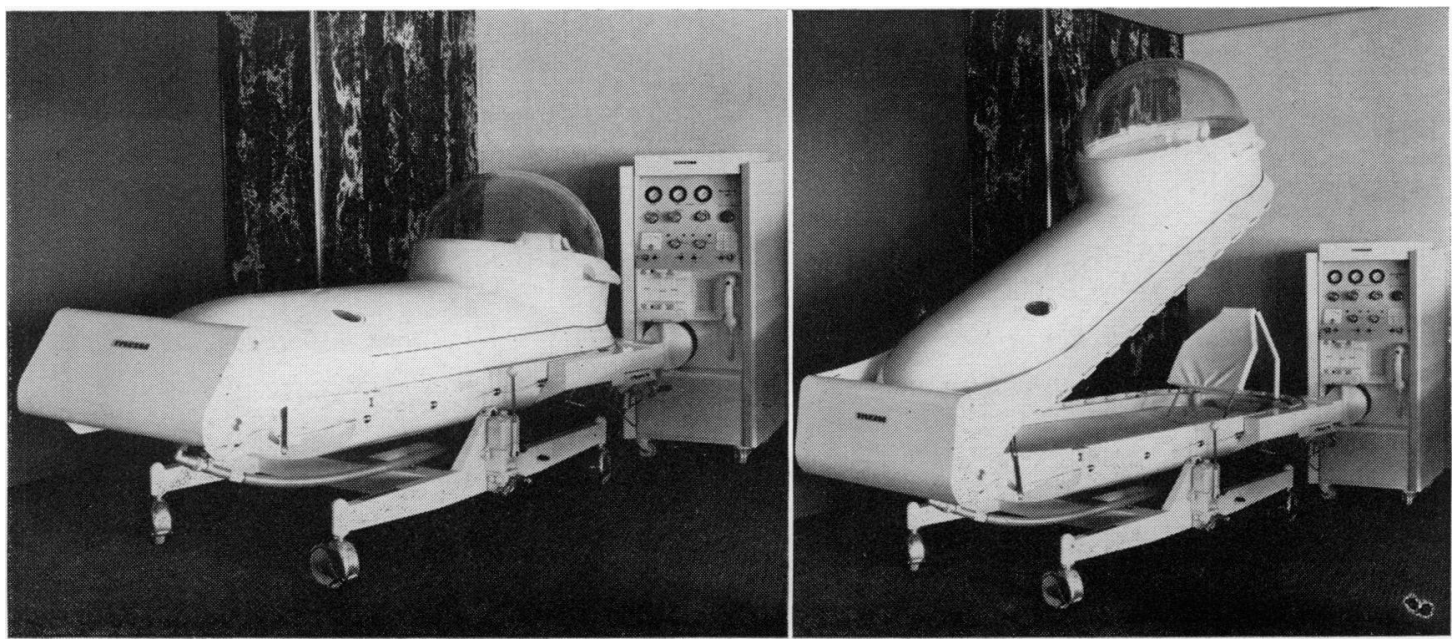

Fig. 1. The hyperbaric oxygen bed.

chamber by flexible tubing. Oxygen is drawn from the hospital piped supply at $60 \mathrm{lb} / \mathrm{in}^{2}$. A regulator allows additional oxygen to enter the line from a reserve cylinder near by, which is automatically drawn upon if the mains pressure falls to $45 \mathrm{lb} / \mathrm{in}^{2}$. This is needed only occasionally so that the reserve cylinder lasts for several weeks.

Oxygen enters the console through a high velocity jet, which creates suction by the Venturi effect. This negative pressure is used to suck the whole gas volume through the system. The maximum consumption of oxygen, on flushing, is about $2501 / \mathrm{min}$, but when pressure is being maintained the amount is between 15 and $30 \mathrm{l} / \mathrm{min}$.

Oxygen enters the chamber through wide slots at the head, behind the back-rest. The flow is directed upwards and sweeps under the surface of the dome, around the patient's head, before escaping through a large-bore pipe at the foot. Exhaled gas is, therefore, at lowest concentration at the head end. A slight draught around the head and shoulders helps to dispel feelings of confinement.

Gas from the chamber then passes to the console, where a sample is continuously bled off to a $\mathrm{CO}_{2}$ analyser. The level is displayed on a large dial on the console. This sampling is done before the gas reaches the soda-lime $\mathrm{CO}_{2}$ absorber. The value displayed is, therefore, the highest concentration in the system. The analyser can be checked for zero at any time by pressing a switch and then returned immediately to analysing the sample gas. The analyser is a katherometer type of $\mathrm{CO}_{2}$ detector and is accurate enough to tell, for example, if the patient is really asleep by the slight fall in $\mathrm{CO}_{2}$ value. Its main use, however, is to monitor the performance of the soda-lime absorber. This is a plastic drum filled with $5 \mathrm{lb}$
$(2 \cdot 2 \mathrm{~kg})$ of soda-lime granules. These change colour from green to brown as they deteriorate and give warning when a change is required. The absorber contains a smaller compartment packed with granules of active carbon. The flow is arranged to pass through the two compartments in sequence, so that 8 carbon dioxide, odours and dust are extracted from the oxygen.

The main flow of reconditioned gas then passes to a heat-exchanger. The first part is cooled by a refrigerating circuit, which causes water vapour of respiration to condense out as water. This trickles down into a reservoir and is subsequently discharged automatically to an external collecting pan. Oxygen is, therefore, prevented from becoming too humid, and is also cooled. The degree of humidity required can be selected on the console. The second part of the heat-exchanger is a heater which rewarms the gas to a temperature which is considered desirable. The heating element is not in direct contact with the oxygen and the interface is not allowed to exceed $50^{\circ} \mathrm{C}$. This is not high enough to ignite dust or particulate matter, even if they had passed through the soda-lime absorber.

The reconditioned oxygen then reaches the suction unit. This employs the Venturi suction from the input jet to maintain the circulation, in a manner similar to a laboratory filter pump. Fresh oxygen mixes with the rest at this point, making up for any loss by leaks and for the amount used by the patient. The selected pressure is thus maintained and any excess escapes via an overflow valve.

The chamber is a largely metal structure weighing $900 \mathrm{lb}(408 \mathrm{~kg})$. The base is made of steel and rests on a chassis with sprung castors and incorporates an axle and hydraulic jack so that the bed can be 
tilted up or down by light pumping of a lever. There is a foam rubber mattress and a back-rest covered with the same material. The lid is made of two layers of light alloy enclosing a sandwich of rigid plastic foam, and incorporates a double-layer Perspex dome at the top and two Perspex windows at the foot. The lid is locked down on to the base by a series of interlocking lugs, these being brought together by an electric motor in $17 \mathrm{sec}$, with manual reversion if the motor fails.

Communication is by telephone at the console and inside the bed by a microphone and loudspeaker built into the base of the dome. The patient, therefore, can hear and speak without having to use a handset. There is also a loudspeaker on the console so that the patient can be heard from elsewhere in the ward. Radio and television sound may be relayed through to the patient, who can see the screen through the transparent dome. The latter facility is important, especially for the longer term patient, as boredom is the only complaint of most of the patients who have been treated.

\section{Patient management}

It is important to explain to the patient the nature of the treatment and description of the apparatus before being taken to it. No special emphasis is put on the considerable rise in pressure, but he is warned about eardrum symptoms and told that these will pass off and that swallowing will help. All pressure changes are made slowly. When the patient is very ill less explanation is necessary, and he often falls asleep shortly after the lid is closed. This is probably due to the isolation from noise which is itself beneficial. Patients never wake up in a panic because they can see the usual surroundings through the Perspex dome as soon as they open their eyes. Care is taken to see that the patients are not in possession of matches or lighters. The risk of fire by static electricity sparks has been made negligible by ensuring that the relative humidity of the circulating gas never falls below $50 \%$.

Having been lifted into the bed, the patient is made comfortable in either the sitting or lying position. If necessary the sphygomomanometer cuff is applied and connected up to the external high pressure supply for inflation. The brachial artery microphone is also strapped in position and plugged into its socket. Three electrocardiograph electrodes are attached to the chest by adhesive patches. This arrangement gives more freedom than when wires are fixed to all four limbs. The patient lead runs to a socket on the base of the bed, and from there continues to an oscilloscope and paper recorder. A radiograph can be taken when the lid is up with the patient in either the sitting or lying position. The $\mathrm{X}$-ray tube is placed under the lid and as far from the patient as possible. Although this distance is much less than usual, satisfactory films are produced. Arterial and venous blood samples are taken in the usual manner between treatments, and a heparinized cannula may be left in situ to save repeated punctures. When an intravenous infusion is running, the plastic container is placed under the buttocks and the weight of the body will maintain the flow. An alternative is to stop the drip before starting the hyperbaric session and then to make up the deficit afterwards. With aqueous solutions the cannula rarely gets blocked and never when heparin is added.

When these preparations are complete, the lid of the bed is lowered. If there is no obstruction at the seal the lid seats down and closes a micro-switch, which turns off a light by the operator and allows the electric closure device to be started. A quiet humming is heard by the patient but no moving parts can be seen. The electric motor stops when closure is complete and the oxygen automatically enters the chamber. This is the flushing phase and, with the circuit open, air is replaced by oxygen for a timed 2 min. A switch is then turned to closed circuit, the required pressure is selected, and the speed of compression adjusted as required.

Some patients have no ear symptoms and can be compressed rapidly, but most have to be watched carefully during the initial phase of compression. The most sensitive patients improve after a few sessions and myringotomy has never been required.

With normal rates of compression full pressure is reached in about $10 \mathrm{~min}$, and the temperature and humidity are adjusted to the patient's comfort. After $1 \frac{1}{2} \mathrm{hr}$ at pressure, decompression is initiated and $10 \mathrm{~min}$ later the lid can be opened to the air. This cycle is repeated as often as is necessary, and this varies from a single session, as for carbon monoxide poisoning, to eight in $24 \mathrm{hr}$ for an acute myocardial infarct. Certain chronic conditions, for example an unhealed wound, may require three sessions a day for 3 weeks.

Nursing care is carried out between sessions. Patients may drink when the lid is down if it is necessary, and male patients can micturate without difficulty, using the usual bottle urinal.

\section{A policy for the use of the hyperbaric oxygen bed}

In our early experience with the prototype model, the problem arose as to the type of patient who should be treated. It was clear that if hyperbaric oxygen was freely available for the treatment of any patient who might benefit from it, much time would elapse before it would be possible to gain any worthwhile experience in the treatment of any one condition. Many patients, such as those with peripheral vascular disease, can be treated with intermittent 
use of the bed, but others, such as those with acute myocardial infarction, may occupy the chamber for up to 3 days, because it is more convenient to nurse them in the chamber, and certainly better for the patients than to move them out of it between sessions of treatment.

It appeared that the types of patients who were most likely to present in reasonable numbers were those with acute myocardial infarction, and it seemed a good policy to devote the chamber primarily to the treatment of such patients, but to use it for other types of cases if time and space permitted. Moreover, the patient with acute myocardial infarction is the type of case which requires to be nursed sitting up.

During the past 2 years we have also been able to use the hyperbaric cylinder, which will accept pressurization up to $3 \mathrm{~atm}$ (atmospheres) absolute compared with 2 atm which is the maximum for the bed. Some of the cases which will be described have had treatment in this cylinder.

\section{Results}

The patients treated fell into fairly well-defined groups. There are too many to describe individually, so the number in each group is given and the overall results are briefly described. The total number of patients treated was 207.

\section{Acute myocardial infarction (forty cases)}

Patients with acute myocardial infarction, which was our main interest, are described in more detail in the next paper (Ashfield \& Gavey, 1969).

\section{Other cardiac conditions (nineteen cases)}

These included acute left ventricular failure, chronic congestive failure, angina and paroxysmal arrhythmias. Angina was usually relieved while in oxygen and the ST segment, if abnormal, reverted to a more normal pattern, but any benefit was confined to the time that the patient was in the oxygen. Acute left ventricular failure was improved and this improvement tended to be maintained. Arterial oxygen values and chest $\mathrm{X}$-rays confirmed this. Congestive heart failure did not respond as well, but here the underlying condition was usually more refractory and was not itself affected by the hyperbaric oxygen. Acute arrhythmias were nearly always corrected.

\section{Unhealed wounds (fifty-nine cases)}

The effect of the high pressure oxygen was easy to assess because the majority of the wounds had been either static for some time or deteriorating. There was a change for the better within 2-3 days of starting treatment. There was no change in those cases where the patient's general condition was hopeless or terminal.

\section{Gas gangrene (eight cases)}

These infections resolved rapidly and there was a reduction in general toxicity. One patient had the infection more than $24 \mathrm{hr}$ kefore treatment started and was not so quickly relieved as the others.

\section{Peripheral ischaemia (twenty-eight cases)}

This group consisted of patients with indolent ulcers, mainly of the lower leg, incipient gangrene and areas with varying degrees of diminished blood supply. The results were good providing the blood supply was not hopeless, and permanent improvement was usually obtained. However, this group required a long course of treatment before any substantial result could be expected.

\section{Cerebral hypoxia (fifteen cases)}

This series comprised patients with low cardiac outputs after surgery, and some had cerebral emboli. The effect of the oxygen was good in the majority of the cases, especially on visual field defects. The effect occurred only when in the chamber, but repeated sessions were used to obtain steady progress. One patient was conscious only when in hyperbaric oxygen, returning to coma whenever he was decompressed. After three sessions he remained conscious in air.

\section{Raynaud's disease (twelve cases)}

These patients were as a rule suffering from systemic sclerosis with chronic involvement of the hands, usually with deep ulcers, when first seen. Hyperbaric oxygen had a remarkable effect on improving the circulation, and all the ulcers healed, except in one case. The increase in warmth and general tissue circulation lasted for many weeks (Copeman \& Ashfield, 1967).

\section{Burns and skin grafts (three cases)}

Observations on these few cases were encouraging.

\section{Carbon monoxide poisoning (thirteen cases)}

One compression only was needed to revive twelve of these patients, who were left with no neurological deficit. One case was virtually dead on arrival and died later, having shown no sign of response when in the hyperbaric oxygen.

\section{Radiotherapy trial (six cases)}

This technique was abandoned because it took too long to transfer the patient to the X-ray machine after giving the hyperbaric oxygen.

\section{The 'bends' (one case)}

The patient was a skin diver who had ascended too rapidly. He had minor neurological signs which 
were much improved after exposure to the high pressure oxygen in a single session. With pure oxygen in the chamber there was no need for lengthy decompression. He had no residual neurological damage on discharge.

\section{Surgical emphysema (one case)}

This patient was treated as a suspected case of gas gangrene, but the final diagnosis was surgical emphysema around an operation wound. He had one treatment only which was very effective.

\section{Paralytic ileus (two cases) \\ Intestinal distension was relieved.}

These cases are quoted principally to demonstrate the wide variety of conditions which might be favourably affected by hyperbaric oxygen therapy.

\section{Conclusions}

The hyperbaric oxygen bed has been used to treat a large number of patients with one of about ten basic diseases. It proved simple to use and reliable in operation. There were no major difficulties in looking after the patients while they received treatment.

The major disadvantage is an inability to get at the patient when the lid is closed for serial observations and other treatments. However, from the beginning it has been possible to measure arterial blood pressure, to record ECG changes and to give intravenous infusions in a rather crude manner, but this situation is bound to improve. At the moment an evaluation is being made of an apparatus to give a more controlled intravenous infusion and of a respirator for use within the chamber, which works on the Coanda principle using a fluid logic system; and also an oxygen tension probe, which would te valuable in giving a continuous measurement of blood and tissue $\mathrm{Po}_{2}$.

Claustrophobia was rarely a problem. The only recurring symptom of patients having a long course was boredom, and this responded, more or less, to radio and television.

Oxygen toxicity was not manifest in any discoverable form. This is undoubtedly due to using the gas at only 2 atm absolute. A further factor was ensuring that carbon dioxide was efficiently absorbed from the closed circuit.

The clinical results were good. Patients with acute myocardial infarcts showed a striking improvement in symptoms and signs, particularly when arrhythmias were present. Pulmonary oedema was relieved in left ventricular failure. Unhealed wounds and various forms of peripheral ischaemia, refractory to other treatment, improved steadily, and for gas gangrene a powerful therapy was available. Carbon monoxide poisoning was relieved and reversed in a single session, failure to respond being due to irreversible brain damage. Cerebral hypoxia responded in certain cases, and the chronic tissue hypoxia in Raynaud's disease showed a surprisingly long-lived improvement. In the surgical field, apart from the unhealed wounds mentioned above, there were promising signs in treating difficult skin grafts, and possibly burns as well. Where recompression is required for any reason the hyperbaric oxygen chamber is probably better than an air chamber, as the inspired gas contains no nitrogen and thus the excretion of this gas from the body is accelerated.

Unfortunately it is very difficult to make an accurate assessment in some cases when those who are administering the treatment have little experience of the disease which is being treated. All that can be said is that the effects are promising and it is obvious that control studies must be made in the case of each disease in order to determine the validity of these clinical impressions. A rapid assessment can only be made if the results are evaluated by experts in the disease, who have access to a large number of patients, rather than those interested primarily in hyperbaric oxygen.

Finally, it must be emphasized that adequate staff, good hospital facilities and constant use of the apparatus are prerequisites for success.

\section{Acknowledgments}

We are grateful to Messrs Vickers Ltd Medical Group for designing, constructing and placing at our disposal the prototype and the latest models of the hyperbaric bed.

Our thanks are due to the nurses and junior medical staff who have looked after these patients, remembering that some of these may be a little frightened by hyperbaric oxygen; and to the Garfield Weston Trust for providing financial assistance, without which this work could not be undertaken.

\section{References}

AshField, R. \& GAVEY, C.J. (1969) Severe acute myocardial infarction treated with hyperbaric oxygen. Report on forty patients. Postgrad. med. J. 45, 648.

Boerema, I., Brummelkamp, W.H. \& Meijne, N.G. (1964) Clinical Application of Hyperbaric Oxygen. Elsevier, Amsterdam.

Copeman, P.W.M. \& Ashrield, R. (1967) Raynaud's phenomenon in scleroderma treated with hyperbaric oxygen. Proc. roy. Soc. Med. 60, 1268. 